andere erkennen lassen. Vier Aufsätze sind es sodann, denen solche Züge ebenfalls zukommen, bei denen aber wissenschaftliche Einzelthemen im Vordergrund stehen. Sie stammen von Doktoranden Simsons. So beschäftigt sich Vorderwiilbecke mit "Verbänden zwischen Gesellschaft und Staat", ein Erfahrungsbericht aus dem Sektor Pharmazie / Arzneimittelherstellung. Fragestellungen der Allgemeinen Staatslehre werden hierbei gesucht. Jansen untersucht das Verhältnis der EU zur WTO vor dem Hintergrund der Rolle der Gemeinschaft in den GATT-Runden. Lippert erörtert Außeneinsätze der Bundeswehr und findet zu Grundfragen der Rolle der Parlamente bei der politischen Willensbildung. Winkelmann stellt die Frage "Reformstau bei der UNO?", auch er zurückgreifend auf persönliche Erfahrungen, hier aus der Tätigkeit bei der Ständigen Vertretung der Bundesrepublik Deutschland bei den Vereinten Nationen. Detailreich und dennoch "theoriebewußt" wird eine Bestandsaufnahme geboten, immer wieder angeleitet durch Gedanken Simsons über die aktuelle und die denkbare Funktion der Weltorganisation.

Schon mit diesen Beiträgen wäre das Buch ein bemerkenswerter Gedenkband. Er gewinnt noch durch die Aufnahme zweier Originalbeiträge des Geehrten. Es sind dies ein ebenso geist- wie humorvoller Vortrag von 1994 über "Die Rolle des Absurden im englischen Denken", im Kern ein Vergleich "englischer" und "deutscher" Verhaltensweisen, nicht bezogen auf Wissenschaftler, aber durchaus auf sie beziehbar, dazu ein längerer Bericht ("Erzählte Erfahrungen"), in welchem von Simson eine nachdenkliche Autobiographie gibt, Eckdaten eines ungewöhnlichen Lebenslaufs aufzeichnet und hierdurch ermöglichte Einsichten wiedergibt. - Ein insgesamt sehr persönliches Gedenkbuch, das aber interessante Lektüre auch für denjenigen enthält, dem es nicht in erster Linie um ein Verständnis des Geehrten geht, sondern der sich allgemein für Zeitgeschichte und die Herausforderungen des Staates, dessen europäische Integration und seine Rolle in der internationalen Gemeinschaft interessiert.

Philip Kunig

\title{
Cornelia Liithy'
}

\section{Verfahren zur friedlichen Beilegung internationaler Streitigkeiten im Rahmen der OSZE}

Schweizer Studien zum Internationalen Recht, Bd. 103

Schulthess Polygraphischer Verlag, Zürich, 1998, 239 S., Sfr. 52,--

In ihrer Arbeit beleuchtet die Schweizerin Cornelia Lüthy das 'Übereinkommen über Vergleichs- und Schiedsverfahren innerhalb der KSZE' (= Stockholmer Übereinkommen) aus dreifacher Perspektive. Zunächst gibt sie eine Übersicht über die bestehenden Prinzipien und Instrumente der friedlichen Streitbeilegung und definiert die der Untersuchung zugrundeliegenden Begriffe, wobei die junge Autorin auch die verwandten Gebiete der 
Politologie und Soziologie zu Rate zieht. Der zweite Teil des Buches ist die Innensicht aus der Perspektive der OSZE, die Analyse ihrer Entwicklung. Aus dem Blickwinkel der Schweiz ist der dritte Abschnitt geschrieben. Hier erklärt die Verfasserin, wie sich das Stockholmer Übereinkommen in das Netz der bestehenden Verpflichtungen auf dem Gebiet der friedlichen Streitbeilegung einfügt.

Als größte Neuerung sieht das Stockholmer Übereinkommen ein obligatorisches Vergleichsverfahren für alle Streitigkeiten vor. In Verbindung mit anderen Streitbeilegungsmechanismen bildet es ein Instrument der OSZE zur Erfüllung ihrer friedenssichernden Aufgabe als Regionalorganisation im Sinne von Kapitel VIII UNO-Charta. Obwohl nur locker in das OSZE-System eingebunden, beurteilt es mit Hilfe seiner Vergleichskommission Streitigkeiten auch aufgrund von OSZE-Verpflichtungen, wodurch diese 'einmahnbar und verfestigt' werden. Trotzdem sieht Cornelia Lüthy das Übereinkommen in Struktur und Verfahren eher den klassischen allgemeinen Streitbeilegungssystemen ähnlich an, "als den insbesondere auf europäischer Ebene geschaffenen organisationseigenen Rechtsschutzsystemen (...)" (S. 234), ein Unterschied, der auch durch die umfassende Subsidiarität des Stockholmer Übereinkommens deutlich wird.

Besonderes Augenmerk sollte der Leser auf den letzten Abschnitt des Buches richten, in dem das Übereinkommen aus Sicht der Schweiz, sozusagen unter ihrer Vorreiterrolle dargestellt wird. Es bleibt kein Platz für die detaillierte Analyse des tatsächlichen Anwendungsbereichs in jedem Mitgliedsland (S. $191 \mathrm{ff}$.), es werden aber der Grundtenor vieler Staaten angesprochen und gute Anleitungen für eigene weiterführende Forschung gegeben. Das Buch ist knapp formuliert. Die Zusammenfassung am Ende ist sehr hilfreich. Der Ansatzpunkt der Arbeit ist interessant, insgesamt kann man sie als gut und lehrreich bewerten, da sie nicht mit zu vielen historischen Vergleichen belastet und durch die in Blockschrift überschriebenen Abschnitte in leicht lesbare 'Portionen' unterteilt ist.

Dagmar Reimmann

\author{
Karine Lescure / Florence Trintignac \\ International Justice for Former Yugoslavia \\ The Working of the International Criminal Tribunal of The Hague \\ Kluwer Law International, The Hague, 1996, 200 pp., $£ 56,00$
}

On May 25, 1993 the Security Council voted unanimously to establish an international tribunal "for the sole purpose of prosecuting persons responsible for serious violations of international humanitarian law committed in the territory of the Former Yugoslavia (...)" (S/Res 827 (1993)). The creation as an ad-hoc tribunal under Chapter VII of the Charter of 